\title{
Plasmonic band gap cavities on biharmonic gratings
}

\author{
Askin Kocabas, ${ }^{1}$ S. Seckin Senlik, ${ }^{1}$ and Atilla Aydinli ${ }^{1,2, *}$ \\ ${ }^{1}$ Department of Physics, Bilkent University, 06800 Ankara, Turkey \\ ${ }^{2}$ Institute of Materials Science and Nanotechnology, Bilkent University, 06800 Ankara, Turkey
}

(Received 6 April 2008; published 30 May 2008)

\begin{abstract}
In this paper, we have experimentally demonstrated the formation of plasmonic band gap cavities in infrared and visible wavelength range. The cavity structure is based on a biharmonic metallic grating with selective high dielectric loading. A uniform metallic grating structure enables strong surface plasmon polariton (SPP) excitation and a superimposed second harmonic component forms a band gap for the propagating SPPs. We show that a high dielectric superstructure can dramatically perturb the optical properties of SPPs and enables the control of the plasmonic band gap structure. Selective patterning of the high index superstructure results in an index contrast in and outside the patterned region that forms a cavity. This allows us to excite the SPPs that localize inside the cavity at specific wavelengths, satisfying the cavity resonance condition. Experimentally, we observe the formation of a localized state in the band gap and measure the dispersion diagram. Quality factors as high as 37 have been observed in the infrared wavelength. The simplicity of the fabrication and the method of testing make this approach attractive for applications requiring localization of propagating SPPs.
\end{abstract}

DOI: 10.1103/PhysRevB.77.195130

PACS number(s): 73.20.Mf

\section{INTRODUCTION}

Collective oscillations of electrons coupled to light form surface plasmon polaritons ${ }^{1}$ (SPPs) that are confined to metal-dielectric interfaces and propagate along the interface with a decaying intensity due to the complex dielectric function of metals. Their small mode volume and sensitivity to the effective index makes SPPs attractive for many applications. ${ }^{2}$ In addition to the already proven biosensing applications, ${ }^{3}$ other diverse possibilities such as increased luminescence efficiency from nanocrystal emitters, ${ }^{4}$ nanolithography, ${ }^{5}$ and nanophotonic applications ${ }^{6-8}$ are being studied. In contrast to propagating modes associated with bound electron plasmas, localized SPPs on small metal particles or voids ${ }^{9-12}$ also attract much attention due to its enhanced fields ${ }^{13}$ particularly for applications such as surface enhanced Raman scattering. ${ }^{14,15}$ Such localized modes are bound to curved surfaces of metal particles characterized by the size and shape of dependent discrete states with various frequencies. The possibility of localizing propagating modes have lately also been attracting interest. ${ }^{16-18}$ Theoretical approaches show that propagating SPPs can also be localized through the use of a properly designed cavity that supports these modes. ${ }^{19}$ Very recently, progress in the theoretical analysis of such cavities has been confirmed with the demonstration of a grating based cavity using Bragg mirrors. ${ }^{17}$ Unfortunately, this approach just allows the mapping of the electric field distribution through the cavity. Direct observation of the cavity formation from plasmonic band structure has still been lacking. Cavities for propagating SPP modes can also be constructed through the selective index loading of grating based SPP systems. In this approach, part of the grating system corresponding to the cavity is left untouched while an extra layer of high dielectric material is deposited onto the grating that is outside the cavity. The induced effective index contrast between the cavity and its surroundings results in the localization of propagating modes ${ }^{20}$ much like those that are supported by Bragg mirrors. ${ }^{17,19,21,22}$ Addition- ally, our approach allows to extract extensive information on the band structure of the cavity and the dispersion of the cavity state in a very simple way for both preparation and characterization of the cavity structure.

The design of such cavities starts by taking into account the dispersion of SPPs on a metallic surface. Dispersion of the SPPs is determined by the dielectric functions of the metal and dielectric environment and results in the SPP wave number being greater than that of the propagating light in the same dielectric medium. ${ }^{1}$ Use of a grating coupler is one way to compensate for the momentum mismatch between the light and the SPP, and excite the SPPs on metallic surfaces. ${ }^{23}$ In this work, we employ grating structures to couple to the plasmons, as well as to form the plasmonic band gap. ${ }^{24}$ The cavity is formed by selective index loading of the grating structures.

\section{EXPERIMENT}

A uniform grating with fixed periodicity was used to couple light to the plasmons. In order to form a plasmonic band gap structure at the wavelength of excitation, additional periodicity needs to be used. This leads to the formation of a biharmonic grating structure. Figure 1(a) shows the AFM image of the biharmonic grating structure used for plasmonic excitation. ${ }^{25,26}$ Gratings with two different periods were successively recorded on a photosensitive polymer (AZ1505) by holographic double exposure interference lithography [Fig. 1(c)]. Figure 1(b) shows a line profile of the biharmonic grating, including two different grating components with periods of $\Lambda_{1}=1330 \mathrm{~nm}$ and $\Lambda_{2}=665 \mathrm{~nm}$. Once developed, the structure is transferred on a photocurable epoxy (OG 146 Epoxy Technology) by a nanoimprint technique with a soft elastomeric mold. ${ }^{27}$ The replication procedure can be summarized as follows; the biharmonic master grating template was prepared using interference lithography, which is used to make the elastomeric stamp. Liquid polydimethylsiloxane (Sylgard 184, Dow Corning) was poured on the master grat- 
a)

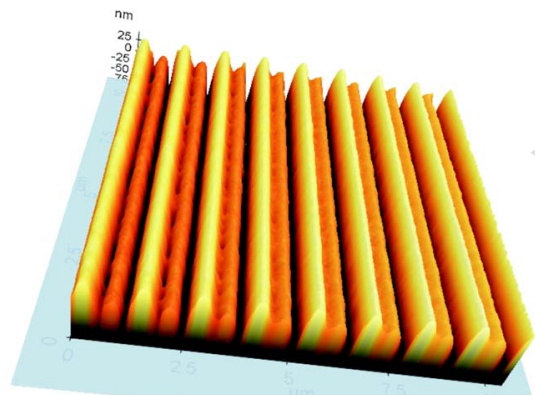

b)

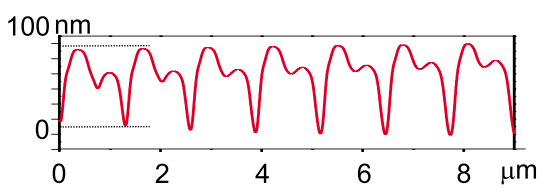

c)

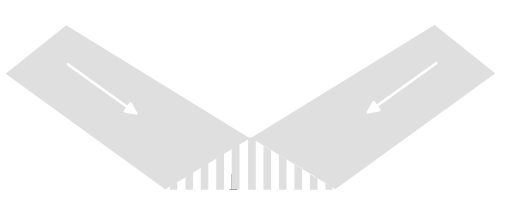

d)

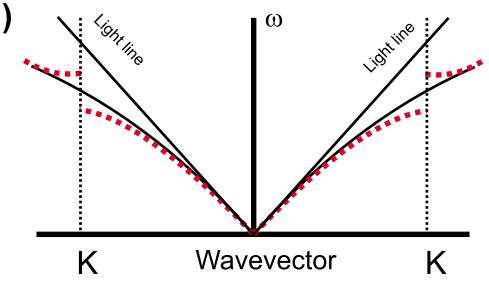

WMV

WMMOMMMM

WhMHMS e)

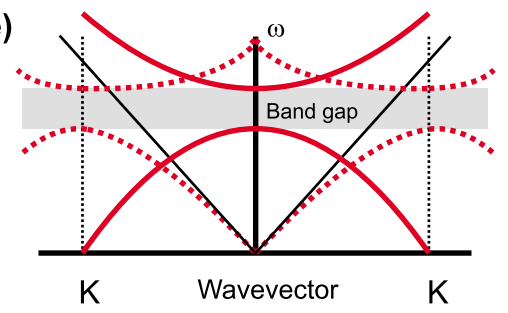

FIG. 1. (Color online) (a) Two-dimensional AFM image of biharmonic metallic surface. First, the periodicity is designed to couple free space light to SPPs. The second one generates the backscattering for propagating SPPs and opens up a photonic band gap. (b) Line profile of the AFM image. (c) Schematics of the double exposure method for grating fabrication. (d) The surface plasmon dispersion curves on a flat metal surface and on uniform grating. A band gap opens up in the case of grating. Dispersion curve lies outside the light line. (e) The surface plasmon dispersion curves on a biharmonic grating. Dispersion with a larger grating component shows the band gap within the light line.

ing and cured at $75^{\circ} \mathrm{C}$ for $2 \mathrm{~h}$. After the curing procedure, the elastomeric stamp was peeled off from the master grating and placed on the prepolymer (OG146) coated wafer. The prepolymer was cured using UV light exposure. Finally, the elastomeric stamp was mechanically removed from the wafer. The photocurable epoxy is chemically inert for the latter optical lithography processes. A 50-nm-thick silver (Ag) film was evaporated on the cured epoxy to form a metallic periodic structure. The long periodicity $\left(\Lambda_{1}\right)$ allows coupling between the incoming photon and the trapped SPPs propagating in forward and backward directions. The SPP coupling is observed at a specific wavelength and the incidence angle, satisfying the following equation:

$$
k_{\mathrm{spp}}=n_{\mathrm{eff}} k_{0}=k_{0} \sin (\alpha) \pm m \frac{2 \pi}{\Lambda}
$$

where $k_{\text {spp }}$ and $n_{\text {eff }}$ are the wave number and effective refractive index of the SPPs. $k_{0}, \alpha$, and $\Lambda$ are the wave number of the incident photon, angle of incidence, and periodicity of the grating structure, respectively. The dispersion curve of SPP on a flat metal surface lies outside the light line and is unable to measure [Fig. 1(d)]. However, the long periodicity changes the dispersion and moves the dispersion curve within the light line [see Fig. 1(e)], thus, allowing the measurement of dispersion around the normal incidence. Measurement of the coupling wavelength and angle reveals the dispersion of the SPPs. The dispersion curves of SPP modes are constructed by scanning the incidence angle and measuring the wavelength dependent reflectivity from the surface. In order to construct the band structure, the reflectivity was measured as follows: collimated white light generated by a Fourier transform infrared (FTIR) spectrometer (TENSOR 37) was directed onto the sample. The back reflected beam from the sample was collected using a beam splitter and was detected with an InGaAs photodetector. The reflectivity mea- surements were taken with different incidence angles from which two dimensional reflectivity maps were constructed. A finite set of measurements were interpolated using linear interpolation algorithm with MATLAB.

Figure 2 shows the wavelength dependent reflectivity map of different grating structures as a function of the incidence angle. The corresponding grating structures are schematically shown on top of each dispersion curve. In Fig. 2(a), each band represents the forward and backward propagating SPPs on a uniform $\left(\Lambda_{1}=1330 \mathrm{~nm}\right)$ grating structure. Uniform grating compensates for the momentum mismatch and acts as a coupler. The second order diffraction from the uniform grating is very weak; hence, SPPs on these types of periodic structures do not show a gap in the dispersion at the excitation wavelength. We note that the minigaps seen in the dispersion curve are due to the discreteness of measurements and the sharp resonances do not allow for interpolation. However, the addition of the second periodicity onto the first one results in a biharmonic structure and creates a backscattering on the propagating SPPs and leads to the formation of standing waves on the biharmonic grating structure. Since the SPPs localized on peaks and troughs of the periodic structure have different energies, a band gap opens up between these lower and higher energy bands, which is denoted by $\lambda^{+}$(or $\omega^{-}$) and $\lambda^{-}$(or $\omega^{+}$), respectively. As seen in Fig. 2(b), the biharmonic structure $\left(\Lambda_{1}=1330 \mathrm{~nm}+\Lambda_{2}\right.$ $=665 \mathrm{~nm}$ ) leads to a band gap with a width of $\Delta=57 \mathrm{~nm}$ around the normal incidence. Controlling the properties of the surface of this structure is critical for fine tuning the characteristics of the band gap. We observe that depositing a thin layer of silicon with full coverage significantly changes the effective indices of the SPPs without perturbing the SPP excitation.

It is well known that the field distributions of $\lambda^{+}$and $\lambda^{-}$ branches of SPPs at the band edges show different confinement behavior. ${ }^{25}$ Figure 3 shows the finite difference time domain (FDTD) simulation results for the electric field dis- 


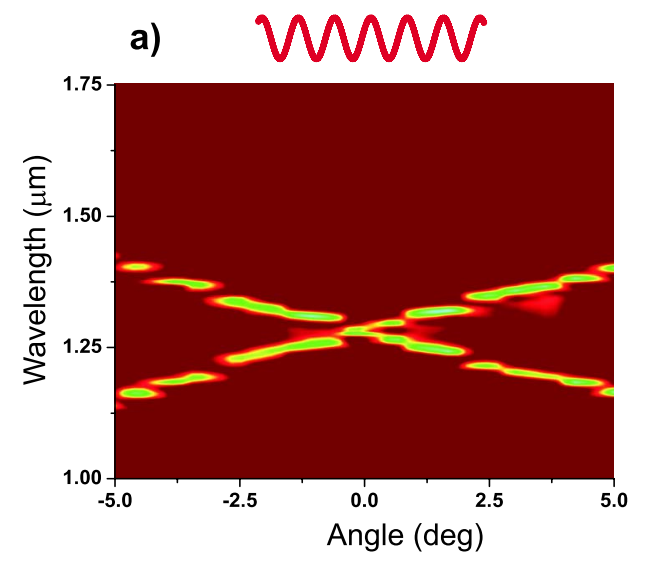

d)

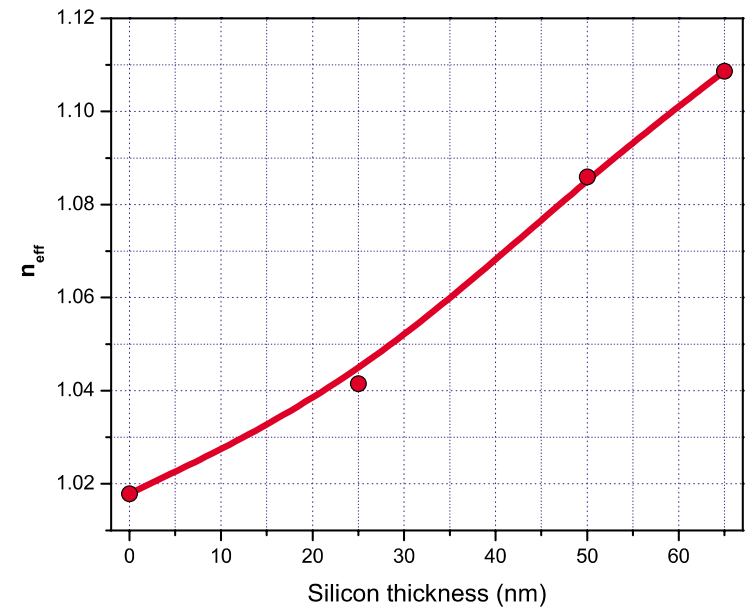

b) MWNWWM

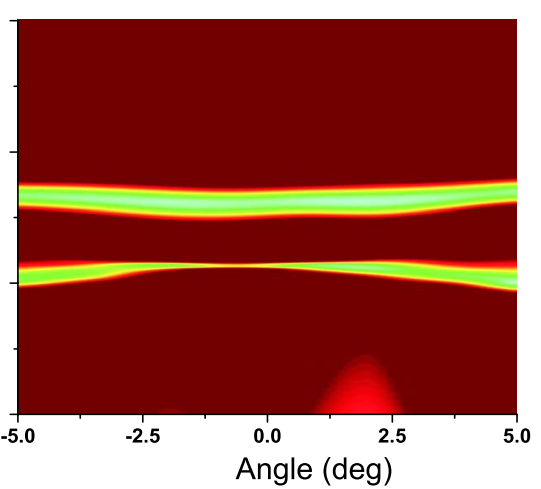

Angle (deg) c) ThWOWH

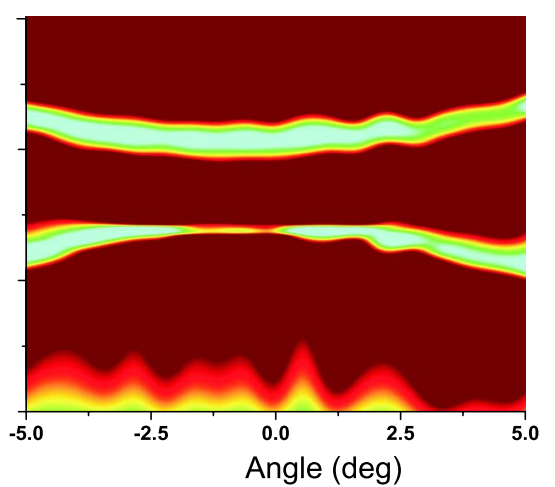

e)

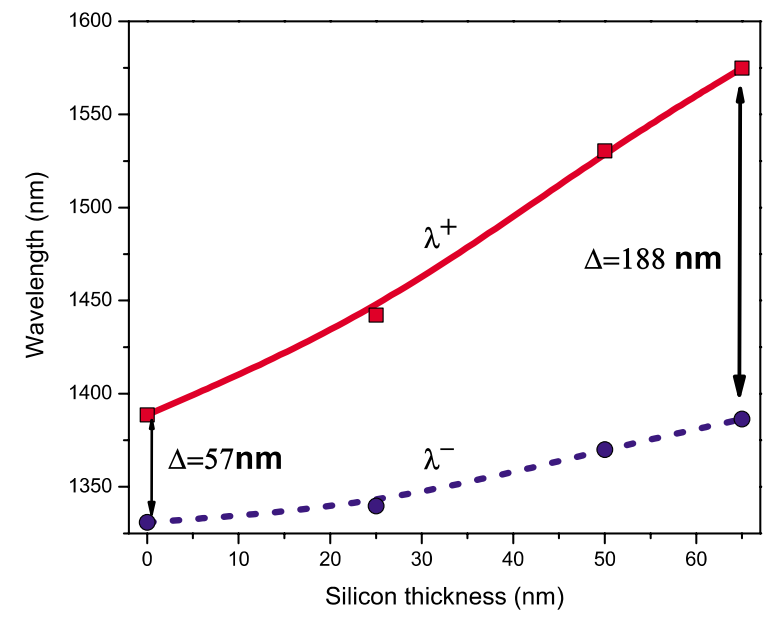

FIG. 2. (Color online) Experimental dispersion curves of SPPs on (a) uniform, (b) biharmonic, (c) silicon-loaded biharmonic metallic surface, and (d) effective refractive indices as a function of silicon thickness. (e) Wavelengths of upper and lower bands $\lambda^{+}$and $\lambda^{-}$as functions of silicon thickness.

tributions on a biharmonic metallic grating structure illuminated with the wavelength of $\lambda^{-}$and $\lambda^{+}$, respectively. $\lambda^{-}$ localizes on the troughs while $\lambda^{+}$localizes on the peaks of the periodic structure. It is seen that $\lambda^{+}$and $\lambda^{-}$show different confinement behavior. The field of $\lambda^{+}$[Fig. 3(b)] becomes more confined to the interface than that of $\lambda^{-}$[Fig. 3(a)]. This extra confinement makes $\lambda^{+}$more sensitive to the surface properties. Thus, loading the biharmonic metallic structure with high dielectric material should increase the energy difference between the $\lambda^{+}$and $\lambda^{-}$bands, which would directly affect the width of the band gap. This reasoning has been tested with the incremental loading of a metallic biharmonic grating structure with silicon $(\mathrm{Si})$. Figure 2(c) shows the dispersion diagram of a biharmonic structure uniformly coated with a 50-nm-thick $\mathrm{Si}$. The $\mathrm{Si}$ coating increases the central wavelength and widens the band gap. We systematically changed the Si thickness and measured the dispersion. Figure 2(d) summarizes these results. There is an increase in effective indices of SPPs as the silicon thickness increases. From Eq. (1) for normal incidence illumination, the effective index of SPPs can be written as $n_{\mathrm{eff}}=\lambda / \Lambda$. Figure 2(d) shows the measured effective indices $n_{\text {eff }}$ of SPPs. The effective index can be tuned from $n_{\text {eff }}=1.02$ to $n_{\text {eff }}=1.11$. Finally, as seen from Fig. 2(e), the deposition of a Si layer increases the width of the band gap up to $\Delta=188 \mathrm{~nm}$. Thus, we demonstrate the ability to systematically control not only the effective index but also the width of the band gap.

On grating structures, forward and backward propagating SPPs having a wavelength in the band gap destructively interfere. However, local perturbation of the effective index can lead to an extra phase change in the propagating waves and can result in a constructive interference at a specific wavelength, which is a cavity mode. Hence, the capability to control the local effective index of SPPs enables the fabrication of plasmonic cavities on grating structures. Therefore, in
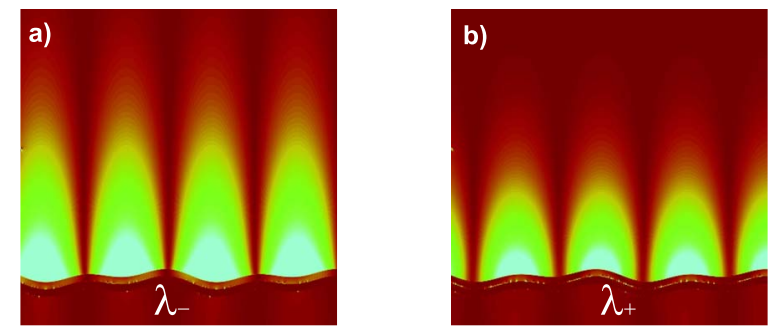

FIG. 3. (Color online) FDTD simulation results for the electric field distributions localized on the biharmonic metallic structure illuminated with wavelengths of $\lambda-$ and $\lambda+$. 

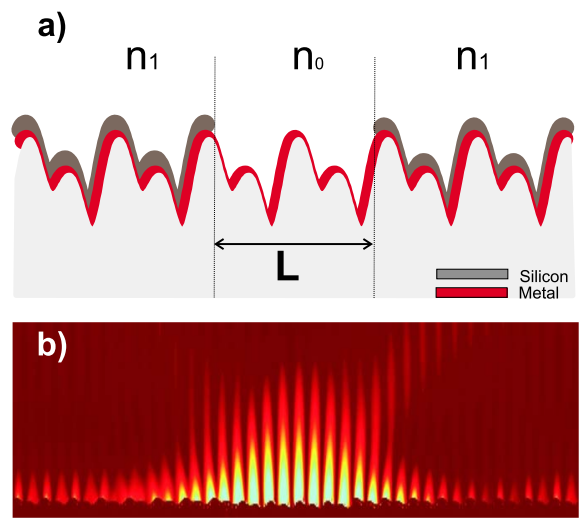

FIG. 4. (Color online) (a) Schematic of the plasmonic cavity structure. (b) FDTD simulation of the electric field distribution in a cavity illuminated with the cavity mode.

the presence of a cavity, SPPs with a previously forbidden wavelength can propagate when the following resonance condition is met: $:^{17,28}$

$$
L \cdot \Delta n=(2 m+1) \frac{\lambda}{4},
$$

where $L$ is the cavity length, $\Delta n$ is the index perturbation, and $\lambda$ is the central wavelength of the band gap. Therefore, we expect to observe a cavity mode at the central wavelength.

A mask with a number of cavities with the same geometry separated by $100 \mu \mathrm{m}$ to prevent a coupling between cavities on both sides in an area of $10 \times 10 \mathrm{~mm}^{2}$, was used to lithographically define the cavity structure on the biharmonic metal surface, and a 65-nm-thick Si was deposited on surface outside the cavity regions. This approach is much simpler than the physical removal of periodicity in a grating. Furthermore, this approach makes use of holographic lithography possible for cavity fabrication, eliminating the need for extensive e-beam writing. Figure 4(a) is a schematic representation of the cavity geometry. From Fig. 2(d), the effective refractive indices of SPPs in silicon-coated and uncoated regions are $n_{0}=1.017$ and $n_{1}=1.108$, respectively. The corresponding effective index difference is $\Delta n=0.091$ and the resulting cavity length for the central wavelength of $\lambda$ $=1.479 \mu \mathrm{m}$ is $L=4.1 \mu \mathrm{m}$.

We performed FDTD simulations to verify the cavity formation. Figure 4(b) shows the simulation result of the electric field intensity $\left(\left|E^{2}\right|\right)$. It is seen that propagating SPPs form a standing wave inside the cavity region. This localization strongly depends on cavity size. According to the results of the simulation, the optimum cavity length was found be around $5.5 \mu \mathrm{m}$, which is slightly larger than that obtained from Eq. (2), which is $4.1 \mu \mathrm{m}$. The difference between the experiment and the simulation results from the small difference between the refractive index of the evaporated $\mathrm{Si}$ and that assumed for the simulation. To clarify the cavity behavior, we measured the dispersion diagram. Figure 5(a) represents the dispersion diagram of the biharmonic grating structure without the cavity and Fig. 5(b) shows the same structure with the cavity. An additional absorption peak ap- pears in the band gap region. Red colored band represents the localized cavity mode. To increase the contrast in the dispersion diagram, the color scale was normalized to $R$ $=80 \%$ and the diffraction background was subtracted from the reflection spectrum. This state shows a nondispersive property. The center of the absorption does not change as a function of the incidence angle, as would be expected from a localized state. ${ }^{11}$ Figures 5(c) and 5(d) show the normal incidence wavelength dependent reflectivity. In Fig. 5(c), two clear absorption peaks correspond to $\lambda^{-}$and $\lambda^{+}$at the band edges. The red arrow in Fig. 5(d) shows additional absorption peak corresponding to the cavity mode. The decrement in the reflected intensity below the $1.3 \mu \mathrm{m}$ is due to the diffraction from the grating structure. Experimentally, we observe the cavity state for a $4.5 \mu \mathrm{m}$ cavity length. The quality factor of the cavity, defined as $Q=\left(\Delta \lambda_{0} / \lambda_{0}\right)$, can be calculated from the reflectivity spectrum. From Fig. 5(d), full width at half maximum of the cavity mode is approximately $\Delta \lambda_{0} \approx 40 \mathrm{~nm}$ and the corresponding quality factor is $Q=37$. This quality factor is relatively low for plasmonic cavities as compared to photonic crystal cavities. The overall quality factor can be described as ${ }^{19}$

$$
\frac{1}{Q}=\frac{1}{Q_{\mathrm{abs}}}+\frac{1}{Q_{\mathrm{rad}}},
$$

where $Q_{\mathrm{abs}}$ and $Q_{\mathrm{rad}}$ are the radiative and absorptive quality factors, respectively. $Q_{\mathrm{abs}}$ is dominated by the metallic loss in the plasmonic cavities and $Q_{\text {rad }}$ by the out-of-plane radiative losses. Gratings that have a larger period component in the cavity region of the biharmonic grating can lead to significant radiative losses. The improvement in quality factor can be achieved by removing the grating structure inside the cavity region. Furthermore, the abrupt change in the localized mode profile in the cavity region is the other radiative loss source. Gently modifying the grating amplitude around the cavity may also suppress the radiation losses. ${ }^{29}$ Gong et al. ${ }^{19}$ proposed the theoretical limit at low temperatures for the quality factor in the case of plasmonic cavities made using Bragg reflectors as 1000 . However, experimental quality factors measured on the cavities made of Bragg mirrors has been reported as $45 .^{17}$

We have systematically studied the formation of plasmonic band gap cavities using numerical calculations. Shown in Fig. 6 are the FDTD simulation results of reflectivity on different grating structures. Figure 6(a) shows the normal incidence wavelength dependent reflectivity of a uniform metallic grating having a periodicity of $\Lambda_{1}=1330 \mathrm{~nm}$. The clear absorption peak corresponds to the plasmonic resonance. Si loading onto the uniform metallic structure increases the effective refractive index of the propagating SPP. This increase in refractive index makes plasmonic resonance shift toward higher wavelengths. Figure 6(b) shows the shifted plasmonic resonance absorption on silicon-loaded uniform metallic grating structures. The second order diffraction from the uniform metallic structure is weak and cannot create a band gap. As seen in Fig. 6(c) superimposing the second grating creates the band gap. Figure 6(c) shows the reflectivity of biharmonic $\left(\Lambda_{1}=1330 \mathrm{~nm}+\Lambda_{2}=665 \mathrm{~nm}\right)$ metallic grating structure. Two resonance peaks correspond to 
a)

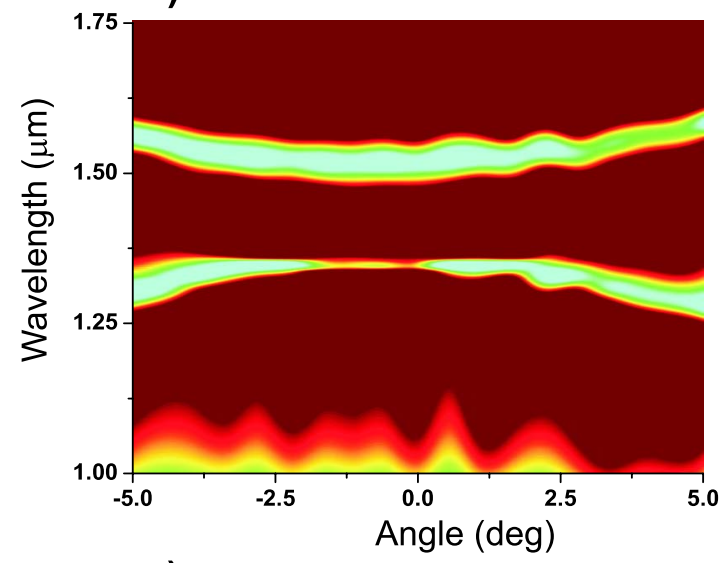

c)

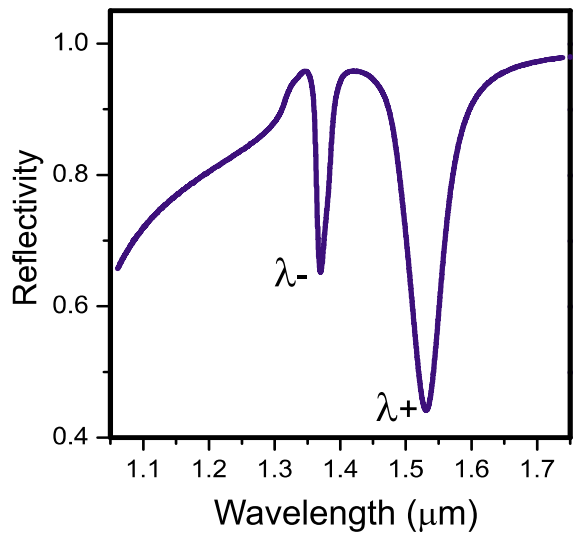

b)

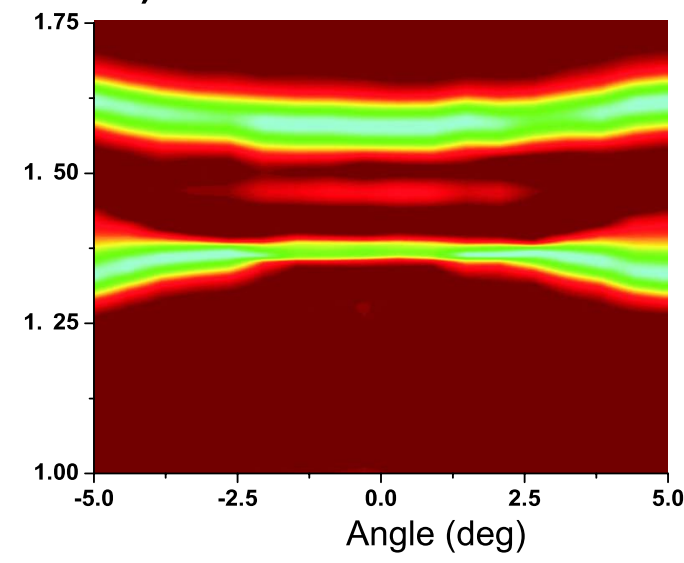

d)

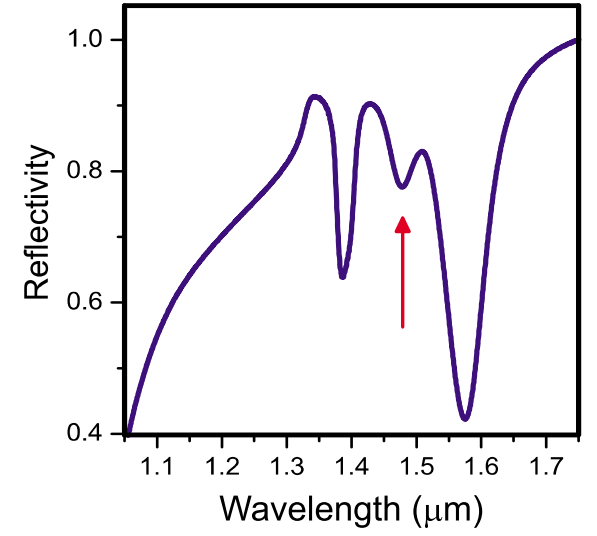

FIG. 5. (Color online) Experimental dispersion curves of SPPs on a biharmonic structure (a) without a cavity, (b) with a cavity, and [(c) and (d)] normal incidence reflectivity spectrum for (a) and (b), respectively.

$\lambda^{-}$and $\lambda^{+}$, respectively. A narrow band gap becomes apparent when the second harmonic component $\left(\Lambda_{2}=665 \mathrm{~nm}\right)$ is superimposed on the grating structure. However, bare biharmonic grating creates a narrow band gap and silicon loading can widen the width of the plasmonic band gap while increasing the effective refractive index. Figure 6(d) shows the reflectivity of the biharmonic $\left(\Lambda_{1}=1330 \mathrm{~nm}+\Lambda_{2}=665 \mathrm{~nm}\right)$ metallic grating structure uniformly loaded with 50-nm-thick Si. Finally, Fig. 6(e) illustrates the reflectivity of the plasmonic cavity fabricated on silicon-loaded biharmonic metallic grating structure. Additional absorption inside the band gap corresponds to cavity resonance.

Many applications such as displays and light emitting devices require the use of the visible part of the spectrum. It would therefore be important to demonstrate that the physical principles and fabrication technology is suitable for the plasmonic cavity formation in the visible, as well. Thus, a plasmonic band gap cavity in the visible regime was designed and fabricated. In this case, the biharmonic structures consisted of gratings with periods of $\Lambda_{1}=500 \mathrm{~nm}$ and $\Lambda_{2}$ $=250 \mathrm{~nm}$ used for coupling and band gap formation, respectively. A spectroscopic ellipsometer (WVASE32) was used to measure the reflection in the visible wavelength range. The normal incidence reflectivity of biharmonic structures [Fig. $7(\mathrm{a})$ ] reveals a wide band gap region in the absence of a silicon layer. To fabricate the cavity, a 12-nm-thick Si load- ing was used for the particular cavity geometry and a $4.5 \mu \mathrm{m}$ cavity length was defined. A plasmonic cavity was obtained in the visible regime; a cavity state appears inside the band gap as expected [Fig. 7(b)]. The effect of the ultrathin layer of $\mathrm{Si}$ on the transmission of light is negligible. The effective refractive index of the SPPs strongly depends on the thickness of the Si loading and restricts systematic studies at visible wavelengths. Uncertainty in our silicon deposition rates makes it difficult to control and the effective indices at the level of the precision are required for a more systematic study. Other materials with high dielectric constants may also be used for the same purpose.

\section{CONCLUSION}

In conclusion, we have used a grating to couple light to the surface plasmons and a second grating superposed onto the first to create a band gap at the excitation wavelength of the first grating. We demonstrated that two superposed grating structures result in a band gap covering the excitation wavelength region. We showed that the band gap widths of grating based plasmonic structures can be controlled with the deposition of high dielectric layers. By selective deposition, we have demonstrated the formation of plasmonic cavities 
a)

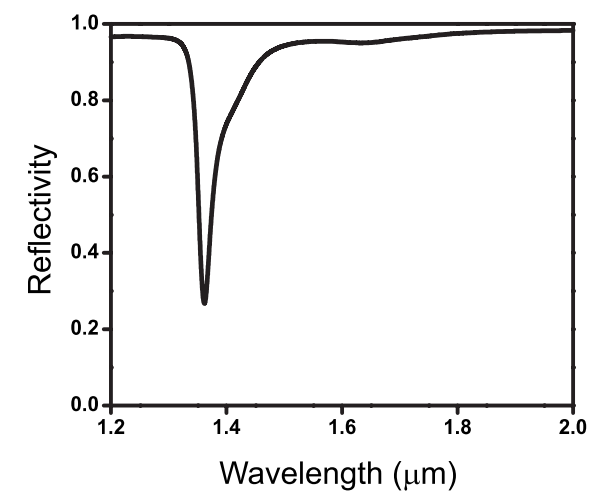

b)

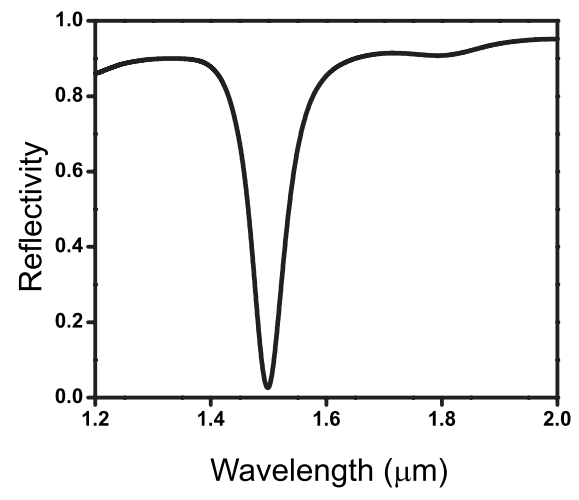

e)

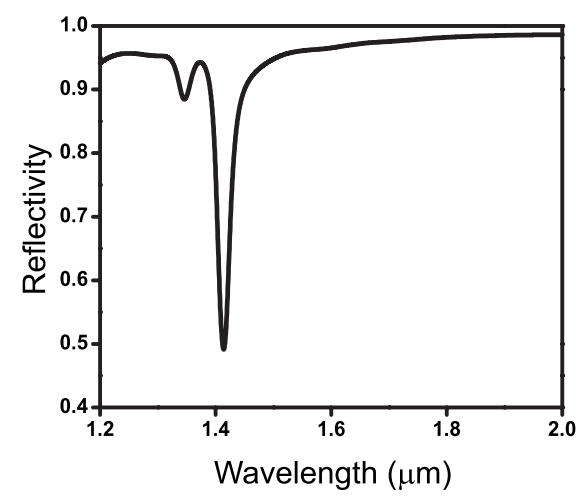

d)

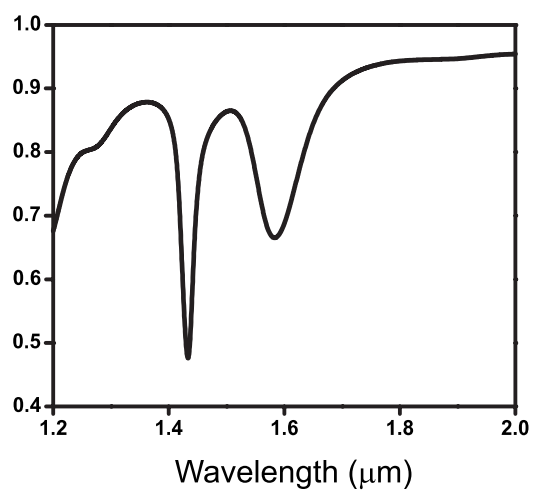

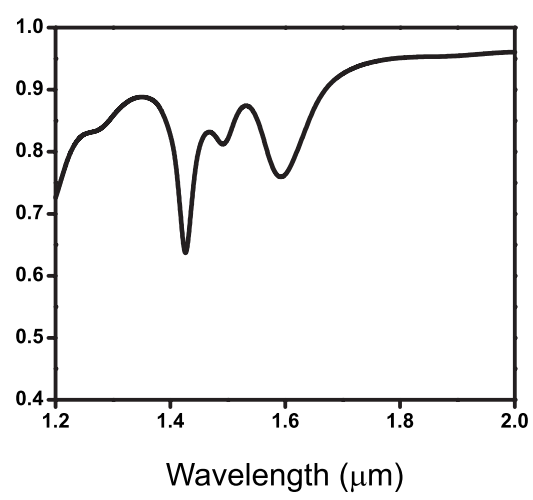

FIG. 6. FDTM simulation results for the reflectivity of different grating structures. (a) Uniform grating $\left(\Lambda_{1}=1330 \mathrm{~nm}\right)$. (b) $50 \mathrm{~nm} \mathrm{Si}$ loaded uniform grating $\left(\Lambda_{1}=1330 \mathrm{~nm}\right)$. (c) Biharmonic metallic grating $\left(\Lambda_{1}=1330 \mathrm{~nm}+\Lambda_{2}=665 \mathrm{~nm}\right)$. (d) Biharmonic $\left(\Lambda_{1}=1330 \mathrm{~nm}+\Lambda_{2}=665 \mathrm{~nm}\right)$ metallic grating structure uniformly loaded with a 50-nm-thick Si. (e) Plasmonic cavity fabricated on siliconloaded biharmonic metallic grating structure.

through the use of biharmonic grating structures with high dielectric superstructures. We observe cavity modes both in the infrared and the visible part of the spectrum. The approach we use is simple, combining holographic lithography

a)

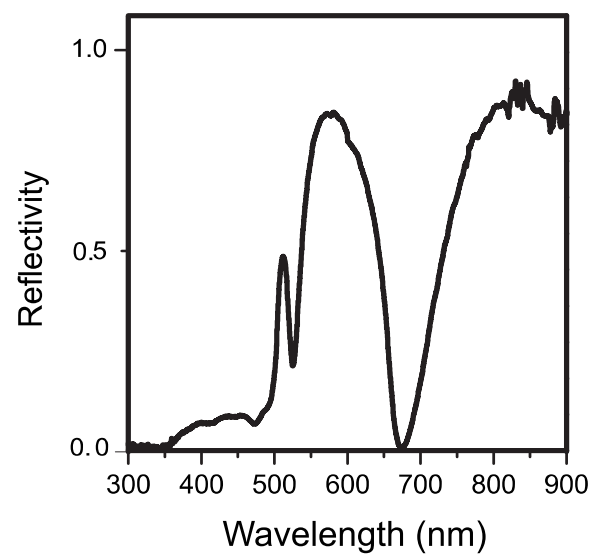

with photolithography to fabricate plasmonic band gap cavities. This approach is suitable for large area applications and it makes plasmonic cavities applicable in examining cavity material interactions. b)

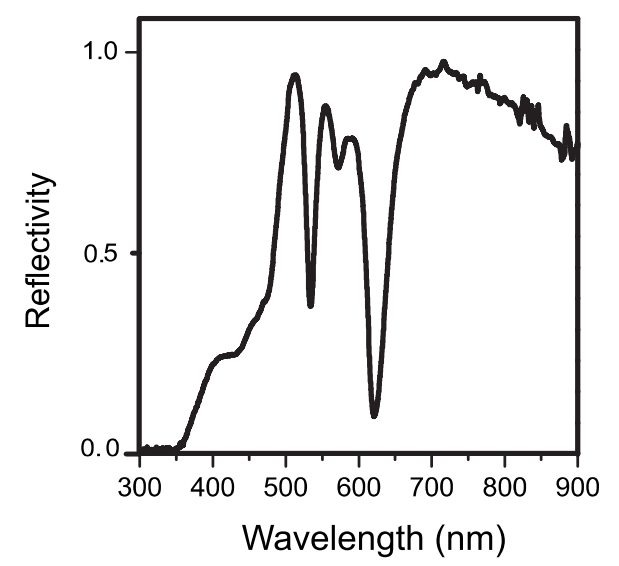

FIG. 7. Normal incidence reflectivity spectrum of (a) the biharmonic and (b) the biharmonic with cavity structure in a visible wavelength range. A cavity state appears inside the band gap region. 


\section{ACKNOWLEDGMENTS}

The authors gratefully acknowledge the financial support of the Turkish Scientific and Technical Research Council
(TUBITAK), Grant No. 104M421, UNAM for the use of the FTIR spectrometer and AFM, A. Dana for his help in AFM measurement and graphics software, and O. F. Ilday for the critical reading of the manuscript.
*Author to whom correspondence should be addressed; aydinli@fen.bilkent.edu.tr

${ }^{1}$ H. Raether, Surface Plasmons (Springer-Verlag, Berlin, 1986).

${ }^{2}$ W. L. Barnes, A. Dereux, and T. W. Ebbesen, Nature (London) 424, 824 (2003).

${ }^{3}$ J. Homola, S. S. Yee, and G. Gauglitz, Sens. Actuators B 54, 3 (1999).

${ }^{4}$ J. S. Biteen, L. A. Sweatlock, H. Mertens, N. S. Lewis, A. Polman, and H. A. Atwater, J. Phys. Chem. C 111, 13372 (2007).

${ }^{5}$ M. Derouard, J. Hazart, G. Lerondel, R. Bachelot, P. M. Adam, and P. Royer, Opt. Express 15, 4238 (2007).

${ }^{6}$ S. I. Bozhevolnyi and V. S. Volkov, Opt. Lett. 26, 734 (2001).

${ }^{7}$ H. J. Lezec, J. A. Dionne, and H. A. Atwater, Science 316, 430 (2007).

${ }^{8}$ W. L. Barnes, J. Opt. A, Pure Appl. Opt. 8, S87 (2006).

${ }^{9}$ T. A. Kelf, Y. Sugawara, R. M. Cole, J. J. Baumberg, M. E. Abdelsalam, S. Cintra, S. Mahajan, A. E. Russell, and P. N. Bartlett, Phys. Rev. B 74, 245415 (2006).

${ }^{10}$ U. Kreibig and M. Vollmer, Optical Properties Metal Clusters (Springer, Berlin, 1995).

${ }^{11}$ N. M. B. Perney, F. J. G. de Abajo, J. J. Baumberg, A. Tang, M. C. Netti, M. D. B. Charlton, and M. E. Zoorob, Phys. Rev. B 76, 035426 (2007).

${ }^{12}$ A. V. Zayats and I. I. Smolyaninov, J. Opt. A, Pure Appl. Opt. 5, S16 (2003).

${ }^{13}$ M. Moskovits, Rev. Mod. Phys. 57, 783 (1985).
${ }^{14}$ K. Kneipp, Y. Wang, H. Kneipp, L. T. Perelman, I. Itzkan, R. Dasari, and M. S. Feld, Phys. Rev. Lett. 78, 1667 (1997).

${ }^{15}$ S. M. Nie and S. R. Emery, Science 275, 1102 (1997).

${ }^{16}$ J. A. Sánchez-Gil, Opt. Lett. 32, 2330 (2007).

${ }^{17}$ J. C. Weeber, A. Bouhelier, G. C. des Francs, L. Markey, and A. Dereux, Nano Lett. 7, 1352 (2007).

${ }^{18}$ B. Wang and G. P. Wang, Appl. Phys. Lett. 87, 013107 (2005).

${ }^{19}$ Y. Y. Gong and J. Vulkovic, Appl. Phys. Lett. 90, 033113 (2007)

${ }^{20}$ J. D. Joannopoulos, R. D. Meade, and J. N. Winn, Photonic Crystals (Princeton University Press, New Jersey, 1995).

${ }^{21}$ J. A. S. Gil, Opt. Express 32, 2330 (2007).

${ }^{22}$ Z. W. Liu, Q. H. Wei, and X. Zhang, Nano Lett. 5, 957 (2005).

${ }^{23}$ R. H. Ritchie, E. T. Arakawa, J. J. Cowan, and R. N. Hamm, Phys. Rev. Lett. 21, 1530 (1968).

${ }^{24}$ S. C. Kitson, W. L. Barnes, and J. R. Sambles, Phys. Rev. Lett. 77, 2670 (1996).

${ }^{25}$ W. L. Barnes, T. W. Preist, S. C. Kitson, and J. R. Sambles, Phys. Rev. B 54, 6227 (1996).

${ }^{26}$ A. C. R. Pipino, R. P. VanDuyne, and G. C. Schatz, Phys. Rev. B 53, 4162 (1996).

${ }^{27}$ A. Kocabas and A. Aydinli, Opt. Express 14, 10228 (2006).

${ }^{28}$ R. C. Alferness, C. H. Joyner, M. D. Divino, M. J. R. Martyak, and L. L. Buhl, Appl. Phys. Lett. 49, 125 (1986).

${ }^{29}$ Y. Akahane, T. Asano, B. S. Song, and S. Noda, Nature (London) 425, 944 (2003). 